\title{
Analysis of Charged-Particle/Photon Correlations in Hadronic Multiparticle Production
}

\author{
T. C. Brooks, M. E. Convery, W. L. Davis, K. W. DelSignore, ${ }^{1}$ T. L. \\ Jenkins, \\ E. Kangas, ${ }^{2}$ M. G. Knepley, ${ }^{3}$ K. L. Kowalski, and C.C. Taylor \\ Department of Physics, Case Western Reserve University, Cleveland, Ohio 44106
}

S. H. Oh and W.D. Walker

Department of Physics, Duke University, Durham, North Carolina 27708-0305

P. L. Colestock, B. Hanna, M. Martens, and J. Streets

Fermi National Accelerator Laboratory, P.O. Box 500, Batavia, Illinois 60510

R. Ball, H. R. Gustafson, L.W. Jones, and M. J. Longo

Department of Physics, University of Michigan, Ann Arbor, Michigan 48109-1120

J. D. Bjorken

Stanford Linear Accelerator Center, Stanford University, Stanford, California 94305

A. Abashian and N. Morgan

Department of Physics, Virginia Polytechnic Institute, Blacksburg, Virginia 24061-0435

C. A. Pruneau

Department of Physics and Astronomy, Wayne State University, Detroit, Michigan 48202

\section{(MiniMax Collaboration)}

\begin{abstract}
In order to analyze data on joint charged-particle/photon distributions from an experimental search (T-864, MiniMax) for disoriented chiral condensate (DCC) at the Fermilab Tevatron collider, we have identified robust observables, ratios of normalized bivariate factorial moments, with many desirable properties. These include insensitivity to many efficiency corrections and the details of the modeling of the primary pion production, and sensitivity to the production of DCC, as opposed to the generic, binomial-distribution partition of pions into charged and neutral species. The relevant formalism is developed and
\end{abstract}


tested in Monte-Carlo simulations of the MiniMax experimental conditions.

\footnotetext{
${ }^{1}$ Now at Department of Physics, University of Michigan, Ann Arbor, MI 48109-1120

${ }^{2}$ Now at Department of Physics, Massachusetts Institute of Technology, Cambridge, MA 02139

${ }^{3}$ Now at Department of Computer Science, University of Minnesota, Minneapolis, MN 55455
} 


\section{INTRODUCTION}

There has recently been renewed interest in semiclassical mechanisms of pion production in high-energy collisions of hadrons and of heavy ions [1-11]. One hypothesis in particular is that pieces of strong-interaction vacuum with an unconventional orientation of the chiral order parameter may be produced in high energy collisions [12]. This disoriented chiral condensate (DCC) is then supposed to decay into a coherent semiclassical pion field having the same chiral orientation.

The primary signature of this mechanism is the presence of large, eventby-event fluctuations in the fraction, $f$, of produced pions that are neutral. Conventional mechanisms of particle production, including those used in standard Monte Carlo simulations, predict that the partition of pions into charged and neutral species is governed by a binomial distribution which, in the limit of large multiplicity, leads to a sharp value of $f \approx 1 / 3$. We refer to this as generic pion production. On the other hand, for the decay of a pure DCC state the distribution of neutral fraction is very different, following an inverse square-root law in the limit of large multiplicity $[1-7,12]$. Some other production scenarios involving the common feature of coherent final states lead to identical $f$ distributions [9, 10, 13-15].

Sophisticated phenomenological techniques have been developed in order to study the properties of multiparticle final states, and much has been done on multiplicity distributions, correlations, and fluctuations [16-20]. Most of the practical studies, however, have considered the properties of a single species at a time. In the case of DCC, formal tools for the study of the joint distribution of neutral and charged pions are required, and here there is much less data and corresponding analysis experience [21-25].

The authors of this paper comprise the MiniMax collaboration (Fermilab T-864), who for the last three years have carried out an exploratory search for signals of DCC at the C0 area of the Tevatron collider [26]. The heart of our detector is a telescope of 24 multiwire proportional chambers (MWPC), with a 1.0 radiation-length lead converter inserted after the eighth plane, so that charged tracks and converted photons can be counted event-by-event. The acceptance in the lego space of pseudorapidity, $\eta$, and azimuthal angle, $\phi$, is roughly a circle of radius 0.65 centered at $\eta=4.1$. In 1995-1996, 8 million triggered events at $\sqrt{s}=1.8 \mathrm{TeV}$ were recorded. The purpose of this paper is not to report the results of this experiment, but rather to describe 
the techniques we are using as the basis of our data analysis strategy. We believe these techniques have much wider applicability and may be of value in other searches for DCC signals.

Even from this very brief description of the experiment, it should be clear that we face many challenges in trying to infer either the presence or absence, within limits, of DCC signals from the data. These include the following:

(a) The MiniMax acceptance is small, so that it is improbable that both $\gamma^{\prime}$ s from a $\pi^{0}$ enter the detector acceptance.

(b) The conversion efficiency per $\gamma$ is about $50 \%$.

(c) Not all $\gamma^{\prime}$ s come from $\pi^{0}$ 's.

(d) Not all charged tracks come from $\pi^{ \pm}$'s.

(e) Because of the small acceptance, the multiplicities are rather low, so that statistical fluctuations are very important.

(f) Detection efficiencies for charged tracks and $\gamma^{\prime}$ 's are momentum-dependent and are not the same.

(g) Efficiency functions may be dependent upon the observed multiplicity or other parameters.

(h) The efficiency for triggering when no charged track or converted $\gamma$ is produced within our acceptance is relatively low and different from that for events in which at least one charged particle or converted $\gamma$ is detected.

Nevertheless, we find that there do exist observables which are robust in the sense that, even in the presence of large (uncorrelated) efficiency corrections and convolutions from produced $\pi^{0}$ 's to observed $\gamma^{\prime}$ s, the observables take very different values for pure DCC and for generic particle production. Each such observable is a ratio, collectively referred to as $R$, of certain bivariate normalized factorial moments, that has many desirable properties, including the following:

1. The $R$ 's do not depend upon the form of the parent pion multiplicity distribution. 
2. The $R$ 's are independent of the detection efficiencies for finding charged tracks, provided these efficiencies are not correlated with each other or with other variables such as total multiplicity or background level.

3. Some of the $R$ 's are also independent of the $\gamma$ efficiencies in the same sense as above. In the remaining cases, the $R$ 's depend only upon one parameter, $\xi$, which reflects the relative probability of both photons from a $\pi^{0}$ being detected in the same event.

4. In all cases $R$ is independent of the magnitude of the null trigger efficiency; see comment (h) above.

5. The ratios $R$ possess definite and very different values for pure generic and pure DCC pion production.

The idealizations implicit in the realization of properties 1-5 include the assumptions that particles other than pions can be ignored, that there is no misidentification of charged particles with photons, and that the production process can be modeled as a two-step process, with a parent-pion multiplicity distribution posited, followed by a particular charged/neutral partitioning of that population by, e.g., a binomial or DCC distribution function. In addition, there is the vital assumption that detection efficiencies for finding a $\pi^{ \pm}$or $\gamma$ do not depend upon the nature of the rest of the event. The validity of these idealizations is not contradicted by the simulations presented in this paper. This idealized model thus appears to be a good basis for a first-order analysis of the properties of the ratios $R$. We anticipate that this will remain true for observations more general than those of the MiniMax experiment.

The layout of this paper is as follows: In Section 2 we review the conventional formalism [16-20] of single-variable generating functions and factorial moments used in describing global multiplicity distributions. We then develop the extensions required to describe the bivariate case of distributions of $\pi^{ \pm}$'s and $\pi^{0}$ 's. The modifications needed to accommodate the decay of $\pi^{0}$ 's into $\gamma$ 's, as well as the inclusion of less-than-perfect detection efficiencies for charged tracks and $\gamma$ 's, are considered in Section 3. In Section 4 we introduce the robust observables $R$ and demonstrate their sensitivity to charged-particle/photon correlations and their insensitivity to detection inefficiencies and the overall aspects of the primary production process for a wide class of production models. The DCC distribution is shown to fall 
into that class, but with distinctly different values of the $R$ 's that clearly distinguish it from the generic distribution under realistic experimental conditions. Generalizations of the formalism which allow for the admixture of both generic and DCC charged/neutral production are considered in Section 5. In Section 6 we estimate, by Monte Carlo simulation as well as by use of the UA5 charged-particle/photon data at $200 \mathrm{GeV}$ and $900 \mathrm{GeV}$ [25], the effects on the $R$ 's from the realistic complications discussed in the preceding paragraph. Concluding remarks are made in Section 7. A number of new results concerning the interpretation and representation of the standard DCC probability distribution that are needed to establish our results concerning DCC production are presented in the Appendix.

\section{GENERATING FUNCTIONS FOR CHARGED- PION/NEUTRAL-PION DISTRIBUTIONS}

The entire content of a set of probabilities $\{P(N)\}$ for the production of $N$ particles in a fixed region of phase space can be encapsulated into the generating function

$$
G(z)=\sum_{N=0}^{\infty} z^{N} P(N)
$$

whose derivatives evaluated at $z=1$ yield the factorial moments

$$
\begin{aligned}
f_{i} & \equiv\left(\frac{d^{i} G(z)}{d z^{i}}\right)_{z=1} \\
& =\langle N(N-1) \cdots(N-i+1)\rangle .
\end{aligned}
$$

It is often useful to express $P(N)$ as a Poisson transform [27] where one introduces a spectral representation in terms of Poisson distributions with a weighting function $\rho(\mu)$ :

$$
P(N)=\int_{0}^{\infty} d \mu \rho(\mu) \frac{\mu^{N}}{N !} e^{-\mu}
$$

where

$$
\int_{0}^{\infty} d \mu \rho(\mu)=1
$$


The Poisson transform isolates the random statistical fluctuations from the physics contained in $\rho(\mu)$. As an example, the negative binomial parametrization

$$
\rho(\mu)=\frac{\lambda^{k}}{\Gamma(k)} \mu^{k-1} e^{-\lambda \mu},
$$

where $\lambda=k /\langle N\rangle$, gives a fairly good two-parameter description of charged multiplicity distributions $[16,17]$. From (1) and (3) we also obtain a spectral representation for the generating function:

$$
G(z)=\int_{0}^{\infty} d \mu \rho(\mu) e^{\mu(z-1)},
$$

where now the factor $e^{\mu(z-1)}$ reflects the purely random character of the Poisson distribution.

The generating function formalism has been widely used to study chargedhadron multiplicity distributions [16-20, 27]. We next generalize this formalism to bivariate distributions of charged and neutral pions. Among our motivations for doing this is the simple manner in which detection inefficiencies and particle decays can be handled with generating functions [27]. These features are particularly important in dealing with the MiniMax experimental situation. Here the parent $\pi^{0}$ 's are not reconstructed from the observed $\gamma^{\prime}$ s and the efficiencies for detecting both the charged particles and the photons are less than perfect. These extensions are taken up in detail in succeeding sections. Some earlier work in this connection is contained in Refs. [21-25]

Let $p\left(n_{c h}, n_{0}\right)$ denote the probability distribution for the occurrence of $n_{c h}$ and $n_{0}$ charged and neutral pions, respectively, in a multiparticle event within a given phase-space region. As in the single-variable case, the content of this bivariate distribution can be conveniently represented by the generating function for factorial moments defined by

$$
G\left(z_{c h}, z_{0}\right)=\sum_{n_{c h}, n_{0}=0}^{\infty} p\left(n_{c h}, n_{0}\right) z_{c h}^{n_{c h}} z_{0}^{n_{0}} .
$$

The partial derivatives of $G\left(z_{c h}, z_{0}\right)$ evaluated at $z_{c h}=z_{0}=1$ generate the factorial moments referring to charged (ch) and neutral (0) particles:

$$
f_{i, j}(c h, 0) \equiv\left(\frac{\partial^{i, j} G\left(z_{c h}, z_{0}\right)}{\partial z_{c h}{ }^{i} \partial z_{0}{ }^{j}}\right)_{z_{c h}=z_{0}=1} .
$$


For example, we have,

$$
\begin{aligned}
& f_{1,0}(c h, 0)=\left\langle n_{c h}\right\rangle, \\
& f_{0,1}(c h, 0)=\left\langle n_{0}\right\rangle, \\
& f_{1,1}(c h, 0)=\left\langle n_{c h} n_{0}\right\rangle, \\
& f_{2,0}(c h, 0)=\left\langle n_{c h}\left(n_{c h}-1\right)\right\rangle .
\end{aligned}
$$

Next, let $P(N)$ be the probability for producing a total of $N$ pions with any distribution of charge among them. Then $p\left(n_{c h}, n_{0}\right)$ can be written as the product of two disjoint probability distributions:

$$
p\left(n_{c h}, n_{0}\right)=P(N) \hat{p}\left(n_{c h}, n_{0} ; N\right),
$$

where $N=n_{c h}+n_{0}$, and

$$
\begin{gathered}
\sum_{N=0}^{\infty} P(N)=1, \\
\sum_{n_{c h}=0, n_{0}=0}^{\infty} \delta_{N, n_{c h}+n_{0}} \hat{p}\left(n_{c h}, n_{0} ; N\right)=1 .
\end{gathered}
$$

What we call the generic model for the charged-neutral distribution $\hat{p}\left(n_{c h}, n_{0} ; N\right)$ involves no correlations, namely, a binomial (Bin) distribution of $n_{c h}$ and $n_{0}$ :

$$
\hat{p}_{B i n}\left(n_{c h}, n_{0} ; N\right)=\left(\begin{array}{c}
N \\
n_{0}
\end{array}\right) \hat{f}^{n_{0}}(1-\hat{f})^{n_{c h}} .
$$

Here $\hat{f}$ is the mean fraction of $\pi^{0}$ 's, which is expected to be about $1 / 3$ as a consequence of isospin symmetry. If we substitute (13) into (10) and explicitly denote the dependence on $\hat{f}$, the generating function (7) becomes, in the binomial case,

$$
G_{B i n}\left(z_{c h}, z_{0} ; \hat{f}\right)=\sum_{N} P(N)\left[\hat{f} z_{0}+(1-\hat{f}) z_{c h}\right]^{N},
$$

which only depends on the linear combination

$$
\zeta \equiv \hat{f} z_{0}+(1-\hat{f}) z_{c h} .
$$

Conversely, if a generating function $G\left(z_{c h}, z_{0}\right)$ is a function only of $\zeta$, the charged and neutral pions are binomially distributed. 
If $P(N)$ is a Poisson distribution, $\ln G_{B i n}\left(z_{c h}, z_{0} ; \hat{f}\right)$ is linear in $\zeta$. The simulations of generic production described in Section 6 yield generating functions that, to good approximation, depend only on a fixed linear combination of $z_{c h}$ and $z_{0}$; the incorporation of the modeling of the MiniMax detector into these simulations is found to alter this linear behavior slightly.

Much of the simplicity of the generic case is also realized for what can be called the binomial transform

$$
\hat{p}\left(n_{c h}, n_{0} ; N\right)=\left(\begin{array}{c}
N \\
n_{0}
\end{array}\right) \int_{0}^{1} d f p(f) f^{n_{0}}(1-f)^{n_{c h}},
$$

of the normalized distribution $p(f)$,

$$
\int_{0}^{1} d f p(f)=1
$$

This leads to a wide class of possible pion factorial-moment generating functions, namely

$$
G\left(z_{c h}, z_{0}\right)=\int_{0}^{1} d f p(f) G_{B i n}\left(z_{c h}, z_{0} ; f\right),
$$

where $G_{B i n}\left(z_{c h}, z_{0} ; f\right)$ is given by (14) with $\hat{f}$ replaced by an arbitrary $f$, $0 \leq f \leq 1$. Combining (3) and (14) we obtain

$$
G\left(z_{c h}, z_{0}\right)=\int_{0}^{\infty} d \mu \rho(\mu) \int_{0}^{1} d f p(f) e^{\mu[\zeta(f)-1]},
$$

where again $\zeta(f)$ is given by $(15)$ with $\hat{f}$ replaced by an arbitrary $f$.

The forms of $p(f)$ and $\rho(\mu)$ depend on the production model and the detector. The uncorrelated, generic case (14) corresponds to $p(f)=\delta(f-\hat{f})$, where $\hat{f}$ is some fixed value of $f$.

It is shown in the Appendix that for a simple DCC model [1-7] and with a sampling prescription appropriate to the experimental situation, $p(f)=$ $1 /(2 \sqrt{f})$. Although the same bivariate distribution is realized in other hadronic production models leading to coherent states [9, 10, 13-15], we refer to this case as the DCC model. We note that in the DCC model $\left\langle n_{0}\right\rangle=2\left\langle n_{c h}\right\rangle$, just as in the generic case for $\hat{f}=1 / 3$.

It is quite possible that the parent pion distribution $P(N)$ or, equivalently, $\rho(\mu)$, will be different for the DCC and generic production mechanisms. This distinction is important for our considerations of admixtures of the two mechanisms. We investigate some possible scenarios for such admixtures in Section 5. 


\section{GENERATING FUNCTIONS FOR CHARGED- PION/PHOTON DISTRIBUTIONS}

For a detector that is designed to observe charged particles and converted $\gamma$ 's within its acceptance, events are classified only according to the numbers of charged particles and photons, $n_{c h}$ and $n_{\gamma}$, respectively. With sufficiently large statistics we can determine probabilities, $p\left(n_{c h}, n_{\gamma}\right)$, for observing these combinations over some portion or all of the available phase space.

In order to obtain the charged-pion/photon generating function, incorporating both $\pi^{ \pm}$and $\gamma$ detection efficiencies from $G\left(z_{c h}, z_{0}\right)$, we extend Pumplin's cluster theorem [27] to the bivariate case. Consider a generating function $G\left(z_{c h}, z_{0}\right)$ that refers to charged and neutral "clusters." Suppose, for the sake of simplicity, the charged clusters decay in a number of ways into charged particles and likewise for the decay of neutral clusters into neutral particles. For each of these decay scenarios there is a probability distribution and a corresponding generating function, $g_{c h}\left(z_{c h}\right)$ or $g_{0}\left(z_{0}\right)$, respectively. The bivariate generating function of the factorial moments of the final chargedneutral particle production is then $G\left(g_{c h}\left(z_{c h}\right), g_{0}\left(z_{0}\right)\right)$. If the charged clusters do not decay, then $g_{c h}\left(z_{c h}\right)=z_{c h}$. On the other hand, $\pi^{0} \rightarrow \gamma \gamma$ with perfect photon detection efficiency correponds to $g_{0}\left(z_{\gamma}\right)=z_{\gamma}^{2}$.

More realistically, there is a probability $\epsilon_{c h}$ for observing a given primary charged pion in the detector and a probability $1-\epsilon_{c h}$ for not observing it. These possibilities can be regarded as the two "decay" modes of the primary charged pion which is otherwise regarded as stable. Similarly, there are probabilities $\epsilon_{m}, m=0,1,2$, with

$$
\epsilon_{0}+\epsilon_{1}+\epsilon_{2}=1,
$$

for observing $m$ photons from a $\pi^{0}$ decay and each possibility can be regarded a decay mode of the $\pi^{0}$ cluster. If these probabilities are identified with what we suppose are the independent, i.e., uncorrelated, efficiencies for the respective detection options, the generating function for the distribution of observed particles, including efficiencies, is obtained from $G\left(z_{c h}, z_{0}\right)$ by replacing $z_{c h}$ by the generating function

$$
g_{c h}\left(z_{c h}\right)=\left(1-\epsilon_{c h}\right)+\epsilon_{c h} z_{c h},
$$


and $z_{0}$ by the generating function

$$
g_{0}\left(z_{\gamma}\right)=\epsilon_{0}+\epsilon_{1} z_{\gamma}+\epsilon_{2} z_{\gamma}^{2}
$$

For the class of production models characterized by (18), the preceding considerations lead to the following factorial-moment generating function for the distribution of observed charged pions and photons:

$$
G_{o b s}\left(z_{c h}, z_{\gamma}\right)=\int_{0}^{1} d f p(f) G_{B i n}\left(g_{c h}\left(z_{c h}\right), g_{0}\left(z_{\gamma}\right) ; f\right) .
$$

The charged-pion/photon factorial moments are

$$
f_{i, j}(c h, \gamma) \equiv\left(\frac{\partial^{i, j} G\left(z_{c h}, z_{\gamma}\right)}{\partial z_{c h}{ }^{i} \partial z_{\gamma}{ }^{j}}\right)_{z_{c h}=z_{\gamma}=1}
$$

which introduces the bivariate indexing $(i, j)$ with respect to charged particles and photons employed henceforth. For example, the two lowest orders of factorial moments are:

$$
\begin{gathered}
f_{1,0}(c h, \gamma)=\left\langle n_{c h}\right\rangle=\langle 1-f\rangle \epsilon_{c h}\langle N\rangle, \\
f_{0,1}(c h, \gamma)=\left\langle n_{\gamma}\right\rangle=\langle f\rangle\left(\epsilon_{1}+2 \epsilon_{2}\right)\langle N\rangle, \\
f_{2,0}(c h, \gamma)=\left\langle n_{c h}\left(n_{c h}-1\right)\right\rangle=\left\langle(1-f)^{2}\right\rangle \epsilon_{c h}^{2}\langle N(N-1)\rangle, \\
f_{1,1}(c h, \gamma)=\left\langle n_{c h} n_{\gamma}\right\rangle=\langle f(1-f)\rangle \epsilon_{c h}\left(\epsilon_{1}+2 \epsilon_{2}\right)\langle N(N-1)\rangle, \\
f_{0,2}(c h, \gamma)=\left\langle n_{\gamma}\left(n_{\gamma}-1\right)\right\rangle=\left\langle f^{2}\right\rangle\left(\epsilon_{1}+2 \epsilon_{2}\right)^{2}\langle N(N-1)\rangle+2 \epsilon_{2}\langle f\rangle\langle N\rangle .
\end{gathered}
$$

In Eqs. (25)-(29) the overall statistical averages for the charged, photon, and charged-photon factorial moments are expressed, in an obvious notation, in terms of the independent moments taken with respect to the $P(N)$ and $p(f)$ distributions.

Finally, we turn to the effect of the MiniMax trigger on these considerations. The MiniMax trigger requires, among other things, a coincidence in the signals from scintillator counters located behind both the converter and the entire tracking telescope. In consequence, events in which no charged particle or converted $\gamma$ goes through the acceptance of the detector are triggered with different (and lower) efficiency, $\epsilon$, than events in which either a charged particle or $\gamma$ conversion products go through the aperture. An effective model 
for the effect of the MiniMax trigger on the probability, $p^{o b s}\left(n_{c h}, n_{\gamma}\right)$, for observing an event with $n_{c h}$ charged particles and $n_{\gamma}$ converted $\gamma$ 's passing through the acceptance is given by the proportionalities

$$
p^{\text {trig }}(0,0)=\epsilon \alpha p^{o b s}(0,0), \quad n_{c h}=n_{\gamma}=0,
$$

and

$$
p^{\text {trig }}\left(n_{c h}, n_{\gamma}\right)=\alpha p^{o b s}\left(n_{c h}, n_{\gamma}\right), \quad n_{c h}+n_{\gamma}>0 .
$$

Here $p^{\text {trig }}\left(n_{c h}, n_{\gamma}\right)$ is the measured probability of seeing an event, including the effects of both the trigger and the particle detection efficiencies, while $p^{o b s}\left(n_{c h}, n_{\gamma}\right)$ presumes perfect triggering. If

$$
\alpha=\left[1+(1-\epsilon) p^{o b s}(0,0)\right]^{-1},
$$

$p^{\text {trig }}$ will be properly normalized if $p^{o b s}$ is.

The bivariate factorial moments transform homogeneously under the transformation (30)-(32) incorporating differential trigger efficiencies,

$$
f_{i, j}(c h, \gamma) \rightarrow \alpha f_{i, j}(c h, \gamma)
$$

\section{ROBUST OBSERVABLES}

The second-order factorial moments (25)-(29) represent the lowest-order correlative effects among charged pions and photons. We see from (29) that the gamma-gamma correlations are distinguished by the term $2 \epsilon_{2}\langle f\rangle\langle N\rangle$ for observing the two photons from a single neutral pion, so that this average will not be a component of a robust measure involving only first and second order moments. This suggests the construction of a measure from the moments (25)-(28) in the form of a ratio in order to cancel out as many effects as possible, apart from the $p(f)$ averages, that reflect the particular details of the production mechanism.

Consider, then, the ratio

$$
r_{1,1}=\frac{\left\langle n_{c h} n_{\gamma}\right\rangle\left\langle n_{c h}\right\rangle}{\left\langle n_{c h}\left(n_{c h}-1\right)\right\rangle\left\langle n_{\gamma}\right\rangle} .
$$

For generating functions of the form (23), we find from (25)-(28) that

$$
r_{1,1}=\frac{\langle f(1-f)\rangle\langle(1-f)\rangle}{\left\langle(1-f)^{2}\right\rangle\langle f\rangle},
$$


an expression in which all reference to the background distribution $P(N)$ and the efficiencies $\epsilon_{1}, \epsilon_{2}$, and $\epsilon_{c h}$ have cancelled out. Further, we see that

$$
r_{1,1} \rightarrow r_{1,1}
$$

under the transformation (30)-(32) so that $r_{1,1}$ is a "robust observable" in the sense referred to in Sec. 1.

It follows from (35) that

$$
r_{1,1} \leq 1
$$

where the equality is realized for generic pion production, $p(f)=\delta(f-\hat{f})$,

$$
r_{1,1}(\text { generic })=1 \text {, }
$$

independently of $\hat{f}$. The realization of the limit (38) in the UA5 data at $200 \mathrm{GeV}$ and $900 \mathrm{GeV}$ [25], and in Monte Carlo simulations at $1.8 \mathrm{TeV}$, both of which include nonpionic sources of charged particles and photons, is considered in Sec. 6.

For a DCC distribution, $p(f)=1 /(2 \sqrt{f})$, one finds

$$
r_{1,1}(D C C)=\frac{1}{2}
$$

This clearly distinguishes the pure DCC and generic distributions.

The values (38) and (39) represent the limiting extremes of a mixture of generic and DCC distributions. Generally, broad (DCC) and narrow (generic) statistical distributions can be distinguished in a mixture of the two by means of higher-order moments that are sensitive to the tail of the charged-particle/photon distribution. Robust combinations of these higherorder moments that are generalizations of $r_{1,1}$ will be of greatest practical value in an analysis of data in which a discernable fraction of DCC form is expected to appear.

Let us first note that the normalized factorial moments

$$
F_{i} \equiv \frac{\langle N(N-1) \ldots(N-i+1)\rangle}{\langle N\rangle^{i}}
$$

are unity if the parent distribution $P(N)$ is Poisson. Therefore, deviations from purely random fluctuations are measured by the departure of the $F_{i}$ 's from unity. A bivariate generalization of the $F_{i}$ 's is given by

$$
F_{i, j}=\frac{\left\langle n_{c h}\left(n_{c h}-1\right) \ldots\left(n_{c h}-i+1\right) n_{\gamma}\left(n_{\gamma}-1\right) \ldots\left(n_{\gamma}-j+1\right)\right\rangle}{\left\langle n_{c h}\right\rangle^{i}\left\langle n_{\gamma}\right\rangle^{j}} .
$$


In particular, one finds that

$$
F_{i, 0}=\frac{F_{i}\left\langle(1-f)^{i}\right\rangle}{\langle(1-f)\rangle^{i}}
$$

and

$$
F_{i, 1}=\frac{F_{i+1}\left\langle f(1-f)^{i}\right\rangle}{\langle f\rangle\langle(1-f)\rangle^{i}}
$$

where $F_{i}$ refers to the $i$ th normalized factorial moment $(40)$ of the $P(N)$ distribution for the total multiplicity. We note that

$$
F_{i, j} \rightarrow \alpha^{1-i-j} F_{i, j}
$$

under the transformation (30)-(32).

Evidently, $r_{1,1}=F_{1,1} / F_{2,0}$. From (42) and (43) we find a generalization of $r_{1,1}$ to a family, $R$, of robust observables:

$$
r_{i, 1}=\frac{F_{i, 1}}{F_{i+1,0}}=\frac{\langle(1-f)\rangle\left\langle f(1-f)^{i}\right\rangle}{\langle f\rangle\left\langle(1-f)^{i+1}\right\rangle} .
$$

Moreover, one finds that for all $i \geq 1$

$$
\begin{aligned}
r_{i, 1}(\text { generic }) & =1 \\
r_{i, 1}(D C C) & =\frac{1}{i+1}
\end{aligned}
$$

in the two cases. Thus, $r_{i, 1}$ becomes more sensitive to the difference between DCC and generic production mechanisms with increasing order of the moments. This reflects the broadness characteristic of the DCC distribution in the neutral fraction $f$ compared to the generic case.

The ratios

$$
r_{i, j}=\frac{F_{i, j}}{F_{i+j, 0}}
$$

are not robust because the moments $F_{i, j}$ for arbitrary $i$ and $j$ are not independent of the photon detection efficiencies. However, the terms involving these efficiencies can be expessed in terms of only one combination of these parameters, namely

$$
\xi=\frac{2 \epsilon_{2}}{\left(\epsilon_{1}+2 \epsilon_{2}\right)\left\langle n_{\gamma}\right\rangle},
$$


along with the mean number of photons, as

$$
F_{i, j}=\sum_{m=0}^{[j / 2]} c_{j, m} \xi^{m} F_{i+j-m} \frac{\left\langle(1-f)^{i} f^{j-m}\right\rangle}{\langle(1-f)\rangle^{i}\langle f\rangle^{j-m}},
$$

The coefficients $c_{j, m}$ are obtained from the identity, true for any differentiable function, $D\left(z^{2}\right)$,

$$
\frac{d^{j} D\left(z^{2}\right)}{(d z)^{j}}=\sum_{m=0}^{[j / 2]} c_{j, m} 2^{m}(2 z)^{j-2 m} \frac{d^{j-m} D\left(z^{2}\right)}{\left(d z^{2}\right)^{j-m}}
$$

The first few $c_{j, m}$ are [28]:

$$
\begin{aligned}
c_{j, 0} & =1, \\
c_{j, 1} & =j(j-1) / 2, \\
c_{j, 2} & =3 j ! / 4 !(j-4) ! .
\end{aligned}
$$

One can use the ratios $r_{i, j}$ 's in the analysis of experimental distributions, with the understanding that the parameter $\xi$ is to be determined from the data. Generally, we have the bounds and limiting values

$$
\begin{gathered}
r_{i, j}(\text { generic }) \geq 1, \\
\left(r_{i, j}(\text { generic })\right)_{\xi=0}=1,
\end{gathered}
$$

and

$$
\left(r_{i, j}(D C C)\right)_{\xi=0}=\frac{i !(2 j-1) ! !}{i+j} .
$$

\section{SENSITIVITY TO DCC ADMIXTURES}

We next turn to the question of what can be said about robust observables when there is an admixture of DCC and generic multipion production. There is considerable theoretical uncertainty about how such an admixture would arise in hadronic collisions and so there are many possibilities for extending the development given in the preceding sections. Our objective in this section is only to provide a formalism in which the sensitivity of experimental results to the presence of DCC or some other anomalous mechanism can 
be investigated. Thus, it will suffice to address this question only in the context of a few simple limiting models of pion production containing both generic and DCC components. Specifically, we consider modifications of the generating-function formalism we have developed in the preceding sections in three different scenarios for mixing DCC and generic multiparticle production. Then we examine the impact of these modifications on the values of the robust observables.

\subsection{Exclusive Production}

First, let us consider the possibility of what we refer to as exclusive production. That is, in a given event, particle production is either the result of the formation of a DCC with probability $\lambda$, or it is generic with binomially distributed charged and neutral particles with probability $1-\lambda$. The picture of exclusive production could be regarded as a first-order phenomenology of very high-energy cosmic-ray interactions, which seem to divide themselves into what appear to be generic and anomalous classes [29].

The generating function for the exclusive production of charged pions and the photons resulting from $\pi^{0}$ decay is simply the weighted sum of the generic and DCC generating functions:

$$
G_{\text {excl }}\left(z_{c h}, z_{\gamma}, \lambda\right)=(1-\lambda) G_{\text {generic }}\left(z_{c h}, z_{\gamma}\right)+\lambda G_{D C C}\left(z_{c h}, z_{\gamma}\right) .
$$

Here $G_{\text {generic }}\left(z_{c h}, z_{\gamma}\right)$ and $G_{D C C}\left(z_{c h}, z_{\gamma}\right)$ are obtained from (23) for the cases $p(f)=\delta(f-\hat{f})$ and $p(f)=1 /(2 \sqrt{f})$, respectively, and where the distributions $P(N)$ of the total number of pions are generally different in the two cases.

The expressions for the moments $r_{i, 1}$ obtained using $G_{\text {excl }}\left(z_{c h}, z_{\gamma}, \lambda\right)$ interpolate between the generic and DCC limits as $\lambda$ varies between 0 and 1 . For example, since

$$
\begin{aligned}
f_{i, j}^{e x c l} & =(1-\lambda) f_{i, j}^{g e n}+\lambda f_{i, j}^{D C C} \\
& =f_{i, j}^{g e n}\left(1+\lambda\left(\frac{f_{i, j}^{D C C}}{f_{i, j}^{g e n}}-1\right)\right),
\end{aligned}
$$

it follows, using the results of Section 3, that one can write

$$
r_{1,1}^{e x c l}(\lambda)=\frac{\left[1+\lambda\left(\frac{2}{15 \hat{f}(1-\hat{f})} \frac{\langle N(N-1)\rangle^{D C C}}{\langle N(N-1)\rangle^{G e n}}-1\right)\right]\left[1+\lambda\left(\frac{2}{3(1-\hat{f})} \frac{\langle N)\rangle^{D C C}}{\langle N\rangle^{G e n}}-1\right)\right]}{\left[1+\lambda\left(\frac{8}{15(1-\hat{f})^{2}} \frac{\langle N(N-1)\rangle^{D C C}}{\langle N(N-1)\rangle^{G e n}}-1\right)\right]\left[1+\lambda\left(\frac{1}{3 \hat{f}} \frac{\langle N)\rangle^{D C C}}{\langle N\rangle^{G e n}}-1\right)\right]}
$$


Note that this expression explicitly depends on the relative size of the DCC and the generic factorial moments. Technically, this ratio is no longer "robust" in the sense of the preceding section. However, it still does not depend upon efficiency corrections. In addition, the extra dependence will be an advantage if DCC dominates the high-multiplicity tail of the distribution.

\subsection{Independent Production}

A second possible production scenario is where the occurrence of DCC in an event is independent of the pions that are produced generically. Independent production implies that the probability $P_{D C C}(N)$ for producing $N$ DCC pions is independent of the probability $P_{\text {generic }}(N)$ for producing $N$ binomially distributed pions, so that the generating function factors into a product,

$$
G_{\text {ind }}\left(z_{c h}, z_{\gamma}\right)=G_{\text {generic }}\left(z_{c h}, z_{\gamma}\right) G_{D C C}\left(z_{c h}, z_{\gamma}\right) .
$$

Thus, we find

$$
f_{i, j}^{\text {ind }}=\sum_{\alpha=0}^{i} \sum_{\beta=0}^{j}\left(\begin{array}{c}
i \\
\alpha
\end{array}\right)\left(\begin{array}{c}
j \\
\beta
\end{array}\right) f_{i-\alpha, j-\beta}^{G e n} f_{\alpha, \beta}^{D C C} .
$$

Hence, using the results of the previous sections, it follows that, for example,

$$
r_{1,1}^{i n d}=\frac{\left[1+\frac{\langle N\rangle^{G e n}\langle N\rangle^{D C C}}{\langle N(N-1)\rangle^{G e n}}\left(\frac{2}{3(1-\hat{f})}+\frac{1}{3 \hat{f}}\right)+\frac{2\langle N(N-1)\rangle^{D C C}}{15 \hat{f}(1-\hat{f})\langle N(N-1)\rangle^{G e n}}\right]\left[1+\frac{2\langle N\rangle^{D C C}}{3(1-\hat{f})\langle N\rangle^{G e n}}\right]}{\left[1+\frac{\langle N\rangle^{G e n}\langle N\rangle^{D C C}}{\langle N(N-1)\rangle^{G e n}}\left(\frac{4}{3(1-\hat{f})}\right)+\frac{8\langle N(N-1)\rangle^{D C C}}{15(1-\hat{f})^{2}\langle N(N-1)\rangle^{G e n}}\right]\left[1+\frac{1\langle N\rangle^{D C C}}{3 \hat{f}\langle N\rangle^{G e n}}\right]} .
$$

Again the sensitivity to the independent production of DCC is dependent on the ratios of DCC and generic factorial moments, but not to the efficiency corrections.

We note that in the independent production model

$$
\ln G_{\text {ind }}\left(z_{c h}, z_{\gamma}\right)=\ln G_{\text {generic }}\left(z_{c h}, z_{\gamma}\right)+\ln G_{D C C}\left(z_{c h}, z_{\gamma}\right),
$$

which suggests an analysis in terms of a bivariate generalization of singlevariable cumulant moments $[16,18-20]$. We define bivariate cumulants for $i+j>0$ by

$$
k_{i, j}=\left(\frac{\partial^{i+j}}{\partial z_{c h}^{i} \partial z_{\gamma}^{j}} \ln G\right)_{z_{c h}=z_{\gamma}=1} .
$$


¿From (58) we see that in this production scenario, the cumulants are additive:

$$
k_{i, j}^{i n d}=k_{i, j}^{\text {generic }}+k_{i, j}^{D C C} .
$$

For single-variable probability distributions, cumulants reflect non-random correlations in that they vanish for a Poisson distribution. In the bivariate case their properties as a measure of correlations are not so direct.

As with the bivariate normalized factorial moments (41), we introduce normalized bivariate cumulant moments:

$$
K_{i, j}=\left\langle n_{c h}\right\rangle^{-i}\left\langle n_{\gamma}\right\rangle^{-j}\left(\frac{\partial^{i+j}}{\partial z_{c h}^{i} \partial z_{\gamma}^{j}} \ln G\right)_{z_{c h}, z_{\gamma}=1} .
$$

In the independent model we obtain for $K_{i, j}^{\text {ind }}$ the weighted sum

$$
K_{i, j}^{i n d}=\lambda_{c h}^{i} \lambda_{\gamma}^{j} K_{i, j}^{D C C}+\left(1-\lambda_{c h}\right)^{i}\left(1-\lambda_{\gamma}\right)^{j} K_{i, j}^{\text {generic }},
$$

where

$$
\lambda_{c h, \gamma}=\frac{\left\langle n_{c h, \gamma}\right\rangle_{D C C}}{\left\langle n_{c h, \gamma}\right\rangle}
$$

are the fractions of the mean charged or photon multiplicities attributed to the DCC.

The formulae for the normalized cumulant moments for DCC and generic subsamples are obtained in a straightforward manner. As before, most of the efficiency corrections cancel out. However, the cumulant moments do not scale homogeneously under the differential trigger inefficiency characteristic of MiniMax. While this is disadvantageous for the early MiniMax analyses, there is reason to expect that they will be eventually of substantial utility in MiniMax as well as in other experiments.

\subsection{Associated Production}

A third possiblity for the contamination of a DCC signal by generic multiparticle production is what can be called associated production. For example, in the Baked Alaska model [8] the number of DCC pions is estimated to scale as

$$
N_{D C C} \sim\left(N_{\text {generic }}\right)^{3 / 2}
$$


A simpler case, which is also a credible scenario, is where the amount of DCC production is, on the average, proportional to the amount of generic production. It then follows using the cluster theorem [27], that

$$
\begin{aligned}
G_{\text {assoc }}\left(z_{c h}, z_{\gamma} ; \lambda\right)= & \int_{0}^{1} d f_{b} p_{b}\left(f_{b}\right) \int_{0}^{1} d f_{d} p_{d}\left(f_{d}\right) \\
& \sum_{N=0}^{\infty} P(N)\left[(1-\lambda) g_{b}\left(z_{c h}, z_{\gamma}\right)+\lambda g_{d}\left(z_{c h}, z_{\gamma}\right)\right]^{N},
\end{aligned}
$$

where,

$$
\begin{aligned}
g_{A}\left(z_{c h}, z_{\gamma}\right) & =f_{A} g_{0}\left(z_{\gamma}\right)+\left(1-f_{A}\right) g_{c h}\left(z_{c h}\right), \\
& p_{b}\left(f_{b}\right)=\delta\left(f_{b}-\hat{f}\right), \\
& p_{d}\left(f_{d}\right)=1 /\left(2 \sqrt{f_{d}}\right),
\end{aligned}
$$

and the index $A$ takes the values $b$ and $d$ in the binomial and DCC cases, respectively. As before, one can carry out the calculation of the robust observables which results in formulae that interpolate as a function of the fraction, $\lambda$, of DCC admixture between the generic and DCC limits. Note that in this case there would be only a single parent $P(N)$, common to both the generic and DCC production. Using the results of the previous section, one can calculate, for example,

$r_{1,1}^{a s s o c}(\lambda)=\frac{\left[(1-\lambda)^{2} \hat{f}(1-\hat{f})+\frac{1}{3} \lambda(1-\lambda)(1+\hat{f})+\frac{2}{15} \lambda^{2}\right]\left[(1-\lambda)(1-\hat{f})+\frac{2}{3} \lambda\right]}{\left[(1-\lambda)^{2}(1-\hat{f})^{2}+\frac{4}{3} \lambda(1-\lambda)(1-\hat{f})+\frac{8}{15} \lambda^{2}\right]\left[(1-\lambda) \hat{f}+\frac{1}{3} \lambda\right]}$,

which, in contrast to the other two cases, (57) and (60), is a fully robust observable.

\subsection{Other Particles}

A similar framework can be used to discuss the sensitivity of the predictions to the production of particles other than pions. This is of potential concern, since $K$ and $\eta^{0}$ production may be a substantial fraction of pion production $[17,25,30]$. In particular, the $\eta^{0} / \pi^{0}$ ratio can be quite large leading to an excess of gammas over the case of pions alone, where $\left\langle n_{\gamma}\right\rangle=\left\langle n_{c h}\right\rangle$.

Relatively little is known about $K$ and $\eta^{0}$ distributions at the highest energies, especially in forward directions, so, while an independent production model might be more accurate, we will limit our considerations at the 
moment to the context of an "associated" production model. In essence, we are thus assuming that a system of parent partons is created in the collision process, and that this system then evolves into a system of $N$ hadrons with probability $P(N)$, with the hadrons independently partitioned into various species.

Let the index $i$ run over the various types of hadrons that are produced. The $i^{\prime}$ th type of hadron is produced with relative probability $\lambda_{i}$ (with $\sum_{i} \lambda_{i}=$ 1 ). These hadrons then decay into charged particles and $\gamma$ 's, and each species of hadron is characterized by a generating function for detecting the products of that species,

$$
g_{i}\left(z_{c h}, z_{\gamma}\right)=\sum_{n_{c h}} \sum_{n_{\gamma}} \epsilon_{n_{c h}, n_{\gamma}}^{(i)} z_{c h}^{n_{c h}} z_{\gamma}^{n_{\gamma}}
$$

where $g_{i}(1,1)=1$. Then the observed generating function, neglecting DCC production, can be written as

$$
G_{o b s}\left(z_{c h}, z_{\gamma}\right)=\sum_{N} P(N)\left[\sum_{i} \lambda_{i} g_{i}\left(z_{c h}, z_{\gamma}\right)\right]^{N}
$$

We can now make a few observations about the impact of contamination of the predictions that arise from $K$ and $\eta^{0}$ production. The following estimates of the effects of various particle types on the magnitude of $r_{1,1}$ draw upon the simulations specific to MiniMax reported in Section 6.

First, we note that the $K^{ \pm}$'s, which are seen simply as charged particles in MiniMax, appear just as another source of charged particles from the collision point and so modify the neutral fraction, but are otherwise benign. Similarly, the $K_{L}$ 's have a decay length much longer on average than the length of the MiniMax detector. In consequence they are only detected, but not identified, when they interact strongly in the converter used to identify photons. On the relatively rare occasions when $K_{L}$ 's do interact in the converter, they are misidentified as $\gamma$ 's. This will also influence the net neutral fraction that is observed, but is also otherwise benign. In conclusion, the associated production of $K^{ \pm}$'s and $K_{L}$ 's will not change the values of the $r_{i, j}$ 's predicted in Section 4 for "generic" production.

The case of $K_{S}$ production is rather interesting since the $K_{S}$ decay modes, $K_{S} \rightarrow \pi^{+} \pi^{-}(69 \%), K_{S} \rightarrow \pi^{0} \pi^{0}(31 \%)$, are essentially those of an isosinglet DCC with one pair of pions. That is, in regard to the statistics of the particles produced, $K_{S}$ decays are essentially identical to those of the smallest 
conceivable domain of DCC's. As such, $K_{S}$ production is in principle of interest from the point of sensitivity to very small domains of DCC.

Let us consider associated production of $K_{S}$ 's with fraction $\lambda_{K_{S}}$. The generating function for studying the modification of generic production is thus

$$
G_{K_{S}}\left(z_{c}, z_{\gamma} ; \lambda_{K_{S}}\right)=\sum_{N} P(N)\left[\left(1-\lambda_{K_{S}}\right) g_{g e n}\left(z_{c}, z_{\gamma}\right)+\lambda_{K_{S}} g_{K_{S}}\left(z_{c}, z_{\gamma}\right)\right]^{N} .
$$

Using previous methods, one finds that

$$
\left(r_{1,1}^{K_{S}}(\lambda)\right)^{-1}=1+\frac{2\langle N\rangle \lambda_{K_{S}} \epsilon_{2,0}^{K_{S}}}{\langle N(N-1)\rangle\left(\left(1-\lambda_{K_{S}}\right)(1-\hat{f}) \epsilon_{c h}+\lambda_{K_{S}}\left(\epsilon_{1,0}^{K_{S}}+2 \epsilon_{2,0}^{K_{S}}\right)\right)^{2}}
$$

which is manifestly not robust.

$K_{S}$ 's are not DCC domains; they are, rather, particles of well-defined mass and a lifetime such that most of them have decayed before reaching the MiniMax detector, and their decay products have strong correlations and are not vertexed to the collison point. As a consequence, in MiniMax the acceptance for 2 charged pions from a single $K_{S}$ is about $4 \%$. Consequently, the impact of $K_{S}$ production on the MiniMax systematics is expected to be quite small.

One can similarly study the impact of $\eta^{0}$ production on the idealized predictions of Section 4. The $\eta^{0}$ has a wider variety of decay modes and all of the charged particles and $\gamma$ 's from the decays are collision vertexed. Thus $g_{\eta^{0}}\left(z_{c}, z_{\gamma}\right)$ is more complicated, but the calculations follow closely those outlined for $K_{S}$ decays. In addition to having decay modes with more than a single charged particle, there are decay modes with intrinsic charged- $\gamma$ correlations, as well as the charged-charged correlations which entered into the $K_{S}$ analysis. The conclusion is, nevertheless, much the same.

\subsection{Detector Effects}

Finally, we note that the formalism we have developed can be extended to consider contamination due to detector-related effects. For example, in detectors which identify gamma rays by electromagnetic calorimetry, charged hadrons can also be identified as photons when they interact strongly in the calorimeter. For example, in WA98 [31], a heavy-ion experiment at CERN 
which has instituted a DCC search, this is expected to occur approximately $20 \%$ of the time. Such misidentifications can be handled by using an appropriate form of the generating function $g_{i}\left(z_{c h}, z_{\gamma}\right)$. For example,

$$
g_{\pi^{ \pm}}\left(z_{c h}, z_{\gamma}\right)=\epsilon_{0,0}^{\pi^{ \pm}}+\epsilon_{1,0}^{\pi^{ \pm}} z_{c h}+\epsilon_{1,1}^{\pi^{ \pm}} z_{c h} z_{\gamma}
$$

would be suitable if some fraction $\epsilon_{11}^{\pi^{ \pm}}$of the charged pions were tagged as both charged particles and photons because of the calorimeter's response.

\section{ROBUST OBSERVABLES IN PRACTICE}

We now turn to the utilization of the robust observables for analyzing collider data, both actual or simulated. As we saw in the last section, the assumptions made earlier are idealizations that are violated by some types of production mechanisms and by less than ideal detector performance. In this section we examine the properties of the robust moments in the context of the UA5 data and Monte-Carlo simulations of the MiniMax detector in order to assess the importance of these violations in practice.

\section{1 $\quad r_{1,1}$ from UA5}

For collider energies of $200 \mathrm{GeV}$ and $900 \mathrm{GeV}$, UA5 measured the inclusive charged-particle and photon $d N / d \eta$ distributions, as well as the corresponding charged-charged and the charged-photon correlation functions, $C_{c h, c h}\left(\eta_{c h}=0, \eta_{c h}\right)$ and $C_{\gamma, c h}\left(\eta_{\gamma}=0, \eta_{c h}\right)$, respectively [25]. Here, $\eta_{c h}$ and $\eta_{\gamma}$ denote the charged-particle and photon pseudorapidities, respectively. The measurements were carried out over about four units of $\left|\eta_{c h}\right|$. The mean values $\left\langle n_{c h}\right\rangle$ and $\left\langle n_{\gamma}\right\rangle$ can be calculated for different pseudorapidity bins using the experimental $d N / d \eta$ distributions. Under the assumption that $C_{c h, c h}\left(\eta_{1}, \eta_{2}\right)$ and $C_{\gamma, c h}\left(\eta_{1}, \eta_{2}\right)$ depend only on the absolute value of $\left|\eta_{1}-\eta_{2}\right|$, the second-order moments that enter into $r_{1,1}$ can be also be calculated for corresponding pseudorapidity bins. Despite large uncertainties in the UA5 photon data and the validity of our assumptions about the correlation functions, we find $r_{1,1}=1.0 \pm 0.10$ for the different energies and various bin choices. 


\subsection{Simulations}

While we believe the robust observables will find general application in experimental searches for DCC, we are motivated here primarily by the MiniMax experimental situation. In this context, in order to make a rough check of the validity of the assumptions we have made in the opening sections, we next describe a series of complete simulations of the MiniMax experiment.

Minimum bias events are generated in PYTHIA version 5.702 and JETSET 7.401 [32]. The output of PYTHIA is then used as input to the simulation of the detector response using GEANT, version 3.21 [33]. The GEANT output is then put through a full tracking and analysis chain. The resulting frequency distributions for observing $n_{c h}$ charged tracks and $n_{\gamma}$ converted photons are then used to calculate the various robust observables. Similar studies, in which the output of PYTHIA is replaced or augmented by the output of a DCC generator, are also carried out. We find the results of these simulations to be in agreement with expectations from our calculations in the previous sections.

\subsubsection{Standard Monte Carlo}

PYTHIA is used to simulate the minimum bias collisions at $\sqrt{s}=1.8 \mathrm{TeV}$. Default values are taken for all parameters except that particles with a mean decay length greater than $1 \mathrm{~cm}$ were not allowed to decay.

There are no published data on multiparticle production at $1.8 \mathrm{TeV}$ in the pseudorapidity interval covered by MiniMax, so there is no independent check on the accuracy of the simulations. For recent measurements at 630 $\mathrm{GeV}$ [34], the agreement between PYTHIA and the $d N / d \eta$ data, in a range of pseudorapidity including that of MiniMax, is less than ideal. Nonetheless, the PYTHIA output represents a useful benchmark.

The particles generated in a simulated collision are then taken as input into a GEANT simulation of the detector and its environment. The experimental data give evidence of a large background of particles aising from interactions in material immediately surrounding the detector. Therefore, many nearby objects are included in the simulation. GEANT propagates the particles through the detector and its surroundings and produces a simulation of the data that are produced by the actual detector. Despite care in including all relevant aspects of the detector and its environment, the GEANT 
data show a smaller number of reporting wires in the MWPC's than do the actual data by a factor of two.

GEANT data are written to a file that is used as input to the same code that is used for the analysis of the actual MiniMax data. The analysis proceeds in two stages. First, a tracking code is used to find track segments in front of (heads) and behind (tails) the converter plane. The output of this calculation is used by a second code (vertexer) that determines the number of charged particles and $\gamma$ 's observed in the event. In so doing, it counts a charged track to be a head that can be joined to at least one tail. A $\gamma$ conversion is taken to be one or more tails emanating from the same point in the converter without an accompanying head. Candidate charged and $\gamma$ tracks are required to point to within some given distance from the collision point in order to remove secondary particles from material adjacent to the detector and fake tracks arising from chance combinations of random report-

ing wires. The parameters used in the vertexer are determined by optimizing the reconstruction of the events generated by PYTHIA and GEANT.

This track-reconstruction procedure is still under development. It does not satisfy all of the assumptions made in Section 1 regarding tracking efficiency. In particular, the reconstruction efficiency may depend on the multiplicity and proximity of tracks.

\subsubsection{DCC generator}

DCC production is modelled according to the $1 /(2 \sqrt{f})$ distribution. For the present simulation, the DCC domain size in $\eta-\phi$ space is taken to be on the order of the detector acceptance. The c.m. momentum of the DCC is directed at the center of the acceptance with a reasonably large $p_{T}$. We assume that the number of pions in the DCC is independent of the central pseudorapidity of the DCC. The ratio of the mean energy density of DCC pions to that of generically produced pions is then approximately constant; we take the ratio to be unity.

DCC's are generated using what could be called a "snowball" model in reference to the low pion momenta in the DCC c.m. The number, $N_{D C C}$, of DCC pions is chosen using a Poission distribution with mean $\mu_{D C C}$.

The neutral fraction is generated using the transformation method, where, if $x$ is a uniform deviate, $f=x^{2}$ is distributed according to $1 /(2 \sqrt{f})$. A uniform deviate $x_{i}$ is then generated for each of the $N_{D C C}$ pions; if $x_{i}<f$, 
the pion is defined to be neutral, otherwise it is defined to be charged. This procedure implements the $1 /(2 \sqrt{f})$ distribution exactly; if one takes the viewpoint that the isosinglet distribution is more fundamental, then this procedure can be viewed as an approximation to it which is valid in the limit that the total number of pions is large, and one is sampling a subset of the DCC. The actual distribution is, of course, an experimental question.

Each of the pions is assigned a 3-momentum in the DCC c.m. system by drawing from a zero-mean Gaussian distribution with a variance $\langle\vec{p} \cdot \vec{p}\rangle=3 \sigma_{p}^{2}$.

The DCC is then boosted such that the momentum of the DCC c.m. is in the direction of the center of the MiniMax detector at $\eta=4.1$, and so that the DCC pions have $\left\langle p_{T}\right\rangle \sim \sigma_{p}$. If the pions are not too relativistic in the DCC c.m. frame, the boosted DCC domain is approximately circular in $\eta-\phi$ space, with radius $R_{D C C} \sim \sigma_{p} / p_{T}$.

The results we report next are based on Monte-Carlo simulations in which $\sigma_{p}=0.1 \mathrm{GeV}$ and $p_{T}=0.14 \mathrm{GeV}$; hence $R_{D C C} \sim 0.7$, the typical radius of a hadronic jet. The Poisson mean for the number of DCC pions has the value $\mu_{D C C}=5.5$, which corresponds to an energy density in lego space comparable to that of generic production. The Monte-Carlo simulation of DCC production is used to generate pure DCC events. These events are then run through the same GEANT simulation as the PYTHIA events, except that the trigger is not used since no particles go in the $\bar{p}$ direction.

\subsection{Results}

Once the number of charged tracks and $\gamma$ 's passing into the acceptance is determined, the moments and $r_{i j}$ are calculated. Statistical errors are estimated assuming Poisson fluctuations and the standard propagation of errors formalism [35].

The results obtained for approximately $5 \times 10^{4}$ PYTHIA events and $2 \times 10^{4}$ pure DCC events are shown in Table 1. For purposes of comparison, the predicted values for idealized binomial and DCC distributions are included. For those ratios involving higher-order moments of the number of observed, converted $\gamma$ 's, the predictions are nonrobust, as discussed in Section 4, and depend on $\xi$, which is determined from the relationship between $f_{0,2}, f_{2,0}$, and $\left\langle n_{\gamma}\right\rangle$, assuming a binomial distribution. The same values are used in correcting the DCC predictions for the higher order moments. In particular, it is assumed that $2 \epsilon_{2} /\left(\epsilon_{1}+2 \epsilon_{2}\right) \approx 0.08 \pm 0.01$ obtained from PYTHIA for 
generic production has the same value for DCC production. This is certainly violated in practice, for the simulated DCC pions have significantly lower

$\left\langle p_{T}\right\rangle \sim \sigma_{p}$ than those generated by PYTHIA, and hence the probablility of both $\gamma^{\prime}$ s from a $\pi^{0}$ decay being in the acceptance, which is reflected in $\epsilon_{2}$, will be different. In addition, the $F_{i}$ 's are also taken to be the same in the DCC case as in the PYTHIA case, which is also clearly a poor assumption. We have chosen to display the data in the manner shown, however, in order to illustrate the problems which will arise in DCC searches using these moments.

There is general agreement between the "predictions" based on the analysis in Section 4 of this paper, and the results of these full simulations. One of the striking features of these results is how well the PYTHIA/GEANT simulation, which includes resonance production, simulations of detector effects, among other features, matches the predictions of a simple binomial model.

In order to illustrate the effect of an admixture of DCC with generic events, where the amount of DCC produced is independent of the amount of generic production, DCC domains from the DCC-generator/GEANT are added to various fractions of random PYTHIA/GEANT events. This represents a mixture of the independent and exclusive models considered in Section 5. The effect on the $r_{i, 1}$ is shown in Table 2 .

These simulations support the expectation that the robust observables introduced in this paper will be a useful analysis tool, even though all of the technical requirements of robustness may not be met. Thus, these observables provide a well-defined framework for describing correlations in such way that many systematic uncertainties cancel out.

\section{CONCLUDING REMARKS}

Most of the experimental analyses and theoretical studies of multihadron production have concentrated only on charged-hadron production, for which the bulk of the data have been taken; for exceptions to this, see [21-25]. The questions we have addressed concerning the neutral-hadron component of multiparticle production have received little attention, but are vital for our MiniMax experiment.

The robust observables $R$ which are here proposed appear, on the basis of the analytic calculations and Monte Carlo simulations we have presented, to be of considerable value in all future analyses of charged-particle/photon 
distributions in high-energy hadron and heavy-ion collisions, and especially with respect to the search for disoriented chiral condensate.

While these observables are manifestly robust, there are still clear limitations to their use which must be eventually be addressed. We have said little about momentum-dependent efficiencies; this will, at the formal level, require generating functions to be generalized to generating functionals [22-24]. At this level, even the choice of the parent generating functional may have considerable ambiguity due to a lack of consensus on the underlying physics; e.g., can the Poisson-transform structure of Eq. (6) be simply generalized?

At a more practical level, the issue of correlated efficiencies, especially with respect to total multiplicity and background level, is vital. Here the features of the individual experiment and its environment are essential, and a strong interplay between simulations and the analysis of real data is required.

Finally, in experiments with large acceptance, even for pure DCC production the chiral order parameter may be different in different portions of the $\eta-\phi$, or lego, phase space. In this case the formalism we have presented must undergo further generalization.

Nevertheless, we believe that the analysis strategy we have described can serve as a very useful starting point for the experimental search for disoriented chiral condensates.

\section{ACKNOWLEDGMENTS}

This work was supported in part by the U.S. Department of Energy, the U.S. National Science Foundation, the Guggenheim Foundation, the Timken Foundation, the Ohio Supercomputer Center, and the Case Western Reserve University Provost's Fund.

\section{APPENDIX: DCC DISTRIBUTIONS}

The distribution

$$
P[n ; N]=\left(\frac{2^{N} N !}{2^{n} n !}\right)^{2} \frac{(2 n) !}{(2 N+1) !},
$$


where $N$ and $n \leq N$ are nonnegative integers, was discovered by Horn and Silver [15] in the context of coherent-state production models. For this reason we will refer to it as the coherent distribution. It was later found that the coherent distribution was an appropriate final state for a simple model of a zero isospin DCC [6]. In both physical contexts, the distribution is relevant to the case of an even total number, $2 N$, of pions and, necesarily, because of zero isospin, to an even number, $2 n$, of $\pi^{0}$ 's. In the mathematical considerations that follow, $n$ and $N$ are regarded as arbitrary nonnegative integers.

In [6] it was shown that

$$
P[n ; N] \rightarrow \frac{1}{2 \sqrt{f}} \frac{1}{N},
$$

as $N, n \rightarrow \infty$, with $n / N \equiv f$ constant, in agreement with the classical expectations for a DCC $[1-5,7,11]$. Generally, a bivariate distribution can be expressed as a continuous binomial distribution weighted over the infinitesampling limit:

$$
P[n ; N]=\left(\begin{array}{c}
N \\
n
\end{array}\right) \int_{0}^{1} \frac{d f}{2 \sqrt{f}} f^{n}(1-f)^{(N-n)} .
$$

The representation (A3) can be established by rewriting (A1) in terms of the beta function,

$$
B(x, y)=\frac{\Gamma(x) \Gamma(y)}{\Gamma(x+y)}
$$

as

$$
P[n ; N]=\frac{1}{2}\left(\begin{array}{c}
N \\
n
\end{array}\right) B\left(n+\frac{1}{2}, 1+N-n\right),
$$

which is found using the identity

$$
\Gamma\left(n+\frac{1}{2}\right)=\frac{(2 n) !}{2^{2 n} n !} \Gamma\left(\frac{1}{2}\right) .
$$

The standard integral representation of the beta function [36] yields (A3). The identity (A5) establishes the connection between the coherent-state production model of Martinis, et al., [9], for $I=0$ and the analysis of [6] and [15]. The continuous binomial distribution (A3) allows one to calculate all averages in the same explicit manner as for the binomial distribution for a 
particular $f$ and then integrate the result over $f$ with the indicated weighting leading to exact results for the various moments. The direct use of (A1) to calculate averages is awkward.

A problem arises with the original interpretation of distribution (A1) in connection with a realistic dectector, or, equivalently, a sampling consisting of a finite number of pions. The limited sampling of such a detector means that typically one sees only a portion of the particular group of the correlated pions that are thought to be the earmark of a DCC. Within that sampling we need to find the distribution induced by the DCC and with it we can carry out a generating-function analysis. We show that the coherent distribution is self-similar in that the neutral/charged distribution of a finite number of pions chosen from a sampling space distributed using the limit of (A1) for $N \rightarrow \infty$, is given in fact by (A1), but now with $N$ and $n$ regarded as the total number and the number of $\pi^{0}$ 's, respectively, whether or not they are even or odd.

In support of these remarks, let us consider the problem of the neutral/charged distribution of an arbitrary subset, even or odd, of a DCC corresponding to $2 N$ pions that are distributed according to $P[n ; N]$. Suppose then that because of limited sampling we observe $n_{t} \leq 2 N$ pions. The joint probability distribution function for finding $n_{0}$ neutral pions and $n_{c h}=n_{t}-n_{0}$ charged pion's is then a product with $P[n, N]$ of the hypergeometric distribution [35] of the two relevant binomial samplings:

$$
Q\left[n_{0} ; n_{t} ; N\right]=\sum_{n \geq \frac{1}{2} n_{0}}^{N-\frac{1}{2} n_{c}}\left(\begin{array}{c}
2 n \\
n_{0}
\end{array}\right)\left(\begin{array}{c}
2(N-n) \\
n_{c h}
\end{array}\right)\left[\left(\begin{array}{c}
2 N \\
n_{t}
\end{array}\right)\right]^{-1} P[n ; N],
$$

where realizing equality in either of the limits is possible only when these limits are even. The nature of the summation limits in (A7) complicates a direct proof of the correct normalization, viz.,

$$
\sum_{n_{0}=0}^{n_{t}} Q\left[n_{0} ; n_{t} ; N\right]=1
$$

however, (A8) has been verified numerically.

Because of the limited sample one cannot regard $N$ in (A7) as known. Therefore the case where all that is known is that $N \gg 1$ is of special interest. 
In this case we find using (A2), the Stirling approximation, and passing to the continuum limit of $f$, that

$$
Q\left[n_{0} ; n_{t} ; N\right] \rightarrow P\left[n_{0} ; n_{t}\right]
$$

where

$$
P\left[n_{0} ; n_{t}\right]=\left(\begin{array}{c}
n_{t} \\
n_{0}
\end{array}\right) \int_{0}^{1} \frac{d f}{2 \sqrt{f}} f^{n_{0}}(1-f)^{\left(n_{t}-n_{0}\right)},
$$

which has precisely the same form as (A3). Here, however, the respective functional parameters are the number of neutral and total pions sampled from the DCC, rather than half those numbers as they are for all of the pions of a full DCC. Thus the induced representation (A10) is a quasi-coherent distribution that goes over to the classical DCC distribution (A2) in in the infinite- $n_{t}$ limit, which in practice may not be too large, due to the accuracy of the Stirling approximation for fairly small numbers.

The similar forms of Eqs. (A3) and (A10) shows that, in regard to an infinite sampling space, the coherent distribution generates a self-similar induced distribution. In addition, the procedure used to arrive at (A10) indicates how one uses the continuum limit of the coherent distribution to define a sampling of a finite number of pions from an infinite sampling space. This remark then also explains the use of the form (A3) when it is applied to the full DCC: It represents a sampling algorithm carried out by means of neutral pairs of pions to induce a DCC of a finite, even number of pions out of the infinite sample.

The distribution (A10) refers to a collection of pions that need not have net zero charge, the signal characteristic of a full DCC, but yet makes no reference to the total charge. For the sampling algorithm used to obtain (A10), the absolute magnitude of total charge will obviously be binomially distributed about zero if $n_{c h}$ is even, and unity if it is not; this extended form of (A10) should be used when the sign of the pions can be distinguished. When they cannot, the means and variances have interpretations that are different from a DCC.

Finally, let us weight $P\left[n_{0} ; n_{t}\right]$ with respect to a parent distribution $P\left[n_{t}\right]$. Then the relevant generating function is

$$
G_{D C C}\left(z_{c h}, z_{0}\right)=\sum_{n_{t}, n_{c h}, n_{0}=0}^{\infty} \delta_{n_{t}, n_{c h}+n_{0}} P\left[n_{0} ; n_{t}\right] P\left[n_{t}\right] z_{c h}^{n_{c h}} z_{0}^{n_{0}} .
$$


Representation (A10) when combined with (A7) yields

$$
G_{D C C}\left(z_{c h}, z_{0}\right)=\int_{0}^{1} \frac{d f}{2 \sqrt{f}} G_{B i n}\left(z_{c h}, z_{0} ; f\right)
$$

which we interpret as the generating function of the factorial moments of the numbers of charged and neutral pions sampled from a very large DCC sample space.

The distribution $p(f)=1 /(2 \sqrt{f})$ has been associated with the decay of a DCC in the classical limit. Thus, the generating function (A12) can be considered applicable to the situation in which the phase-space domain of the particles resulting from the DCC is very much larger than the acceptance

of the detector. Then one can picture DCC production as corresponding to an event distribution for which the neutral fraction $f$ is a random variable distributed according to $1 /(2 \sqrt{f})$, a depiction reflected in (A12).

\section{REFERENCES}

1. A. A. Anselm, Phy. Lett. B217,169 (1989).

2. A. A. Anselm and M. G. Ryskin, Phy. Lett. B266, 482 (1991).

3. J. D. Bjorken, SLAC-PUB-5545, Int. J. Mod. Phys. A7, 4189 (1992).

4. J. D. Bjorken, Acta Phys. Pol. B23, 561 (1992).

5. J-P. Blaizot and A. Krzywicki, Phys. Rev. D 46, 246 (1992).

6. K. L. Kowalski and C. C. Taylor, CWRUTH-92-6, hep-ph/9211282.

7. K. Rajagopal and F. Wilczek Nucl. Phys. B399, 395 (1993).

8. J. D. Bjorken, K. L. Kowalski and C. C. Taylor, SLAC-PUB-6109, Proc. of Les Rencontres de la Vallée D' Aoste, La Thuile, 1993, ed. M. Greco, Editions Frontier, p. 507 (1993). 
9. M. Martinis, V. Mikata-Martinis, A. S̆vorc, and J. Črnugelj, Phys. Rev. D 51, 2482 (1995); hep-ph/9411329; hep-ph/9501210.

10. R. D. Amado et al., Phys. Rev. Lett. 72, 970 (1994).

11. B. Müller, Rep. Prog. Phys. 58, 611 (1995).

12. For a review, K. Rajagopal, in Quark-Gluon Plasma 2, ed. R. Hwa, World Scientific, 1995, HUTP-95-A013.

13. I. V. Andreev, JETP Lett. 33, 67 (1981).

14. V. Karmanov and A. Kudrjavtsev, ITEP-88, 1983.

15. D. Horn and R. Silver, Ann. Phys. (N.Y.) 66, 509 (1971).

16. P. Carruthers and C. C. Shih, Int. J. of Mod. Phys. A 2, 1447 (1987).

17. C. Geich-Gimbel, Int. J. of Mod. Phys. A 4, 1527 (1989).

18. I. M. Dremin, Mod. Phys. Lett. A8, 2747 (1993); Pis'ma Zh. Eksp. Teor. Fiz. 59, 561 (1994) [trans. JETP Lett. 59, 585 (1994)].

19. I. M. Dremin and R. C. Hwa, Phys. Rev. D 49, 5805 (1994).

20. For a review, E. A. DeWolf, I. M. Dremin, W. Kittel, hep-ph/950325.

21. G. H. Thomas and B. R. Webber, Phys. Rev. D 9, 3113 (1974).

22. L. Diósi, Nucl. Instrum. Methods 138, 241 (1976).

23. L. Diósi, Nucl. Instrum. Methods 140, 533 (1977).

24. L. Diósi and B. Lukács, Phys. Lett. B206, 707 (1988).

25. R. E. Ansorge et al., Z. Phys. C 43, 75 (1989).

26. For descriptions and preliminary results of the MiniMax experiment (T-864) see: J. D.Bjorken et al, Fermilab Proposal T-864, April 1993. J. D. Bjorken, K.L. Kowalski, and C. C. Taylor, in Proc. of the 1993 Madison/Argonne Workshop on Physics at Current Accelerators and the Supercollider, ed. J. L. Hewett, A. R. White, and D. Zeppenfeld, 
p. 73, hep-ph/9309235. J. D. Bjorken, SLAC-PUB-6430-1994. C. C. Taylor, in Proc. Inter. Conf. on Elastic and Diffractive Scattering, ed. H. M. Fried, K. Kang, and C-I Tan, World Scientific, 1994, p. 348. C. C. Taylor, in Hot Hadronic Matter: Theory and Experiment, ed. J. Letessier et al., Plenum Press, NY, 1995, p. 503. W. L. Davis et al, in Proc. of the 24th Int. Cosmic Ray Conf., Rome, September 1995. C. C. Taylor, Eialot, 1995. M.E. Convery et al, Bull. Am. Phys. Soc. 41, 902 (1996). W. L. Davis et al, Bull. Am. Phys. Soc. 41, 938 (1996). J. D. Bjorken, proceedings of the Nijmigen workshop, 1996. C. C. Taylor, proceedings of the RHIC workshop, 1996. J. Streets, Bull. Am. Phys. Soc. 1996; Proc. of the DPF meeting, 1996.

27. J. Pumplin, Phys. Rev. D 50, 6811 (1994).

28. I. S. Gradshteyn and I. M. Ryzhik, Table of Integrals, Series, and Products, Academic Press, Orlando, 1980, p. 20.

29. C. M. G. Lattes et al., Phys. Rep. 65, 151 (1980); L.T. Baradezi et al., Nucl. Phys. B370, 365 (1992); S-I Hasegawa, ICR-151-87-5, unpublished.

30. G. Alverson et al., Phys. Rev. D 48, 5 (1993).

31. A. L. S. Angelis et al., CERN/SPSLC/91-17, May 1991; CERN/SPSLC/9535, May 1995, WA98 collaboration. B. Wyslouch, for the WA98 collaboration, talk at ICHEP, July 251996.

32. H. -U. Bengtsson and T. Sjöstrand, Comp. Phys. Comm. 46, 43 (1987).

33. GEANT-Detector and Simulation Tool, CERN, PM0062 (1993).

34. C. J. Liapis, Yale University thesis, May, 1995. CERN P238 Collaboration, J. Ellett et al., to be published.

35. A. G. Frodesen, O. Skjeggestad, and H. Tøfte, Probability and Statistics in Particle Physics, Universitetsforlaget, Bergen, 1979.

36. A.Erdélyi, Higher Transcendental Functions, McGraw-Hill, New York, 1953, Vol. 1, p. 10. 
Table 1. Robust observables $r_{i, j}$ for generic events simulated by PYTHIA and pure DCC events simulated with the 'snowball' model. Comparisions with the $r_{i, j}$ 's obtained with binomially distributed pions and the $1 /(2 \sqrt{f})$ classical limit of DCC's.

\begin{tabular}{cccccc} 
& & PYTHIA & DCC & binomial & $1 /(2 \sqrt{f})$ \\
$i$ & $j$ & $r_{i, j} \pm \sigma_{r_{i, j}}$ & $r_{i, j} \pm \sigma_{r_{i, j}}$ & $r_{i, j}$ & $r_{i, j}$ \\
\hline 1 & 1 & $1.00 \pm 0.02$ & $0.57 \pm 0.01$ & 1.00 & 0.50 \\
2 & 1 & $1.00 \pm 0.05$ & $0.43 \pm 0.03$ & 1.00 & 0.33 \\
3 & 1 & $1.04 \pm 0.13$ & $0.38 \pm 0.05$ & 1.00 & 0.25 \\
0 & 2 & $1.36 \pm 0.04$ & $1.55 \pm 0.06$ & 1.36 & 1.80 \\
1 & 2 & $1.36 \pm 0.10$ & $0.66 \pm 0.06$ & 1.30 & 0.62 \\
2 & 2 & $1.47 \pm 0.26$ & $0.44 \pm 0.09$ & 1.25 & 0.31 \\
0 & 3 & $2.13 \pm 0.25$ & $2.98 \pm 0.39$ & 1.89 & 3.54 \\
1 & 3 & $2.03 \pm 0.43$ & $1.19 \pm 0.31$ & 1.74 & 0.90 \\
0 & 4 & $3.06 \pm 0.94$ & $6.82 \pm 2.18$ & 2.70 & 7.34
\end{tabular}


Table 2. The effect on the $r_{i, 1}$ of an admixture of DCC and generic (PYTHIA) events. DCC domains from the DCC-generator/GEANT are added to various fractions of random PYTHIA/GEANT events. The first column represents the fraction of events in which a DCC is overlaying a generic event.

\begin{tabular}{ccccc} 
DCC fraction & $r_{1,1} \pm \sigma_{r_{1,1}}$ & $r_{2,1} \pm \sigma_{r_{2,1}}$ & $r_{3,1} \pm \sigma_{r_{3,1}}$ & events \\
\hline 0.00 & $1.01 \pm 0.02$ & $1.02 \pm 0.05$ & $1.09 \pm 0.14$ & 51741 \\
0.02 & $1.00 \pm 0.02$ & $1.00 \pm 0.05$ & $1.01 \pm 0.15$ & 51741 \\
0.05 & $0.97 \pm 0.02$ & $0.93 \pm 0.05$ & $0.95 \pm 0.10$ & 51741 \\
0.10 & $0.95 \pm 0.02$ & $0.89 \pm 0.04$ & $0.89 \pm 0.08$ & 51741 \\
0.20 & $0.93 \pm 0.02$ & $0.83 \pm 0.04$ & $0.77 \pm 0.07$ & 51741 \\
0.50 & $0.84 \pm 0.01$ & $0.71 \pm 0.03$ & $0.68 \pm 0.06$ & 40000 \\
1.00 & $0.74 \pm 0.01$ & $0.60 \pm 0.03$ & $0.55 \pm 0.06$ & 20000
\end{tabular}

